\title{
Standard Approach to Gastrointestinal Stromal Tumors - Differences between China and Europe
}

\author{
Lin Tu $^{a}$ Peter Hohenberger ${ }^{b}$ Heike Allgayer ${ }^{c}$ Hui Cao ${ }^{a}$ \\ ${ }^{a}$ Department of Gastrointestinal Surgery, Renji Hospital, School of Medicine, Shanghai Jiao Tong University, Shanghai, China; \\ ${ }^{b}$ Division of Surgical Oncology, Mannheim University Medical Center, Medical Faculty Mannheim, University of Heidelberg, \\ Mannheim, Germany; \\ ${ }^{c}$ Department of Experimental Surgery - Cancer Metastasis, Medical Faculty Mannheim, University of Heidelberg, Mannheim, Germany
}

\section{Keywords}

Gastrointestinal stromal tumor, GIST · Diagnosis .

Guideline $\cdot$ Targeted therapy $\cdot$ Tyrosine kinase inhibitor

\section{Summary}

Gastrointestinal stromal tumors (GISTs) are the most common mesenchymal tumors of the gastrointestinal tract. With the considerable research and application of molecular-targeted therapy for GISTs in the last two decades, GISTs have become a model of multidisciplinary oncological treatment. Although Western clinical guidelines are available for GISTs, such as those by the European Society of Medical Oncology (ESMO), the clinical situations in China are different from those in European countries. There are distinct differences between the clinical practice, diagnostic methods, surgical approach, and availability of new targeted agents in China and those in Europe. This review summarizes the Chinese GIST consensus guidelines compared to the European ones, which may provide an optimal approach to the diagnosis and management of GIST patients.

(C) 2018 S. Karger GmbH, Freiburg

\section{Introduction}

Gastrointestinal stromal tumors (GISTs) are the most common mesenchymal tumors of the gastrointestinal tract with a wide range of biological behaviors from benign to highly malignant [1]. GISTs are believed to arise from the interstitial cells of Cajal (ICC) or their precursors and are histologically heterogeneous, showing spindle cells (70\%), epitheloid cells (20\%), and mixed cell types
(10\%) [2]. The initial event in GIST tumorigenesis is the gain-offunction mutation within the KIT molecule, a member of the receptor tyrosine kinase III family, or platelet-derived growth factor receptor alpha (PDGFRa) receptor tyrosine kinases [3].

GISTs have become a model of multidisciplinary oncological treatment with surgical and medical oncologists, pathologists, gastroenterologists, and radiologists who are the essential participants to provide optimal treatment for GIST patients. A systematic review of population-based cohort studies on GISTs showed that the incidence ranges from a low 0.43 per 100,000/year in the Shanxi Province, China, to a high 1.6-2.2 per 100,000/year in South Korea [4-6]. The cohort of 13,550 patients from 19 countries studied in this review gave the reported age as ranging from 10-100 years, with a median age in the 60s; both male and female populations had approximately equal distribution [7]. The anatomical locations of GISTs are frequently the stomach (55.6\%) and small bowel $(31.8 \%)$, while they are less commonly found in the colon and rectum (6\%), other various locations (5.5\%), and the esophagus (0.7\%) [7].

The incidence of clinical GISTs, GISTs with considerable risk for recurrence, or metastatic and/or recurrent disease, with an estimated unadjusted incidence of around 1/100,000/year, is considered to be no different between Asian and Western countries. Similar to other countries (see contribution by Raut [8] in this issue of VISCERAL MEDICINE), the handling of GISTs needs to be separated from the diagnosis and treatment of gastric cancer. In East Asia, gastric cancer screening sometimes finds an asymptomatic submucosal tumor $<5 \mathrm{~cm}$, which may be pathologically diagnosed as GIST after surgical resection. Thus, the incidence and the relative frequency of gastric GISTs are higher in East Asian countries compared with Western countries, especially for asymptomatic and small gastric GISTs [9].

\section{KARGER}

(c) 2018 S. Karger GmbH, Freiburg 


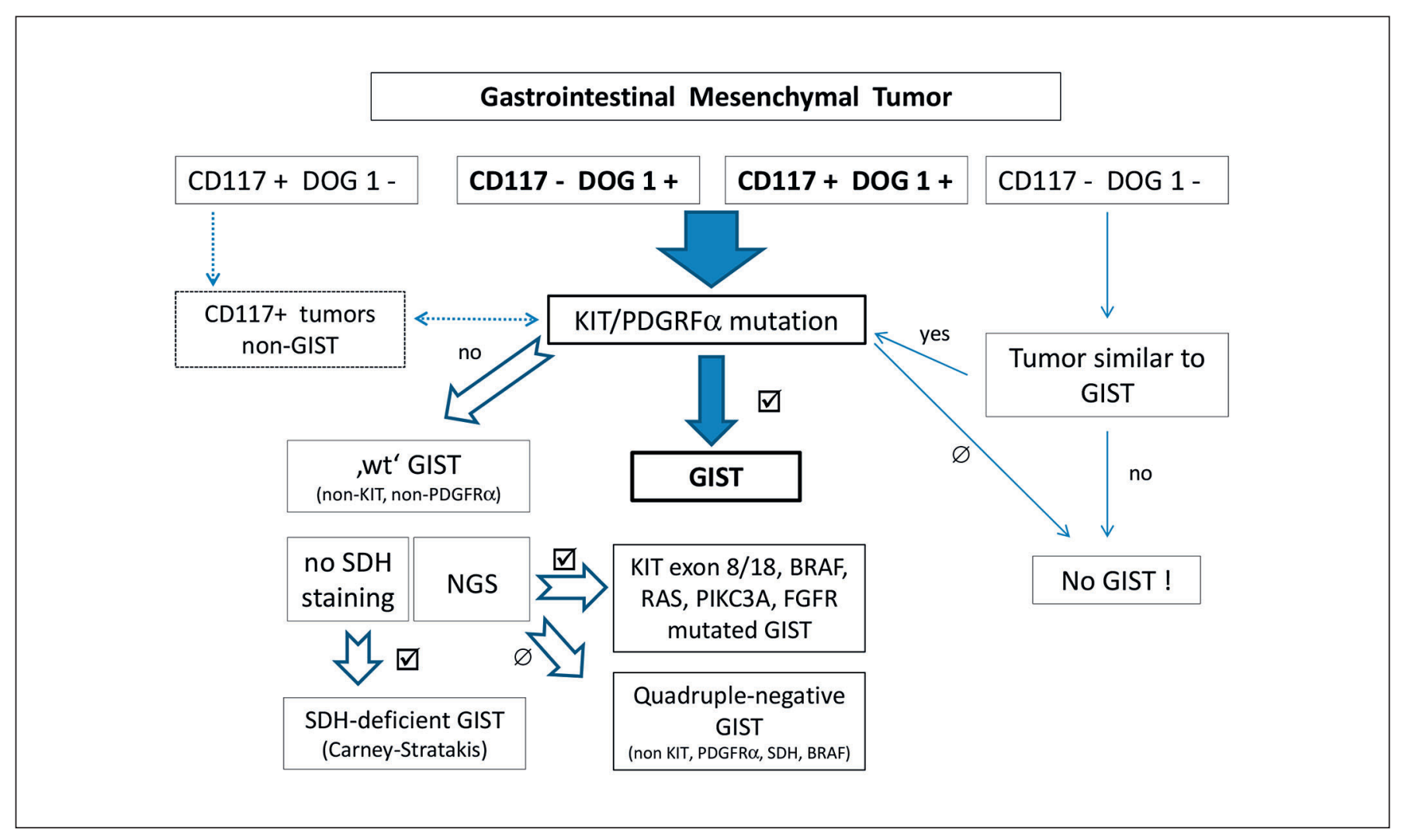

Fig. 1. Pathological diagnostic algorithm of gastrointestinal stromal tumors (GISTs) (modified from [5]).

\section{Clinical Problems of GIST Primary Tumors}

In a recent analysis of the management of hemorrhage of GISTs in China, Liu et al. [10] describe that gastrointestinal bleeding is the most common and the most dangerous complication, often necessitating emergency surgery. The risk of such surgery is significantly higher than that of elective surgery [10]. The authors also focus on the fact that although the mechanism of bleeding in GISTs is not yet fully understood, existing studies suggest that GISTs associated with bleeding tend to indicate a worse prognosis. In the acute hemorrhagic phase, digital subtraction angiography (DSA) is often positive and has hemostatic effects [11]. However, DSA alone cannot distinguish benign from malignant tumors. Definitive diagnosis requires endoscopic ultrasonography (EUS) and pathological examination. GISTs may have 'air sign' on plain film which is caused by internal bleeding, necrosis, or ulceration.

\section{Clinical Management Guidelines}

Clinical guidelines for GISTs were first published by the National Comprehensive Cancer Network (NCCN) in 2004 and by the European Society of Medical Oncology (ESMO) in 2005, both with recent updates in 2018 [12]. Although most evidence and clinical guidelines for the treatment and management of GISTs have been established in Western countries, Asian patients have sup- plied limited data regarding the diagnosis and treatment of GIST. It is unclear whether these guidelines are appropriate for the clinical situation in Asian countries, especially China, given the longlasting experience of selected Chinese centers [13]. The Chinese consensus guidelines for the diagnosis and management of GISTs were formulated in 2013 and updated in 2017, and differ from those of European countries in terms of clinical practice patterns [5]. In this review, we will summarize the clinical practice of both China and European countries and then discuss the similarities and differences between the European and Chinese GIST guidelines, although the fundamental approaches to the diagnostic and treatment strategy for GISTs are very similar.

\section{Pathological Diagnosis and Genetic Analysis}

CD117 and DOG1 are usually positive in immunohistochemical (IHC) staining, and thus are useful to confirm the diagnosis. Most GISTs show differentiation towards ICC and have an activating mutation in the gene encoding KIT or PDGFRa receptor tyrosine kinase. In a few cases where the mutation of KIT or PDGFRA is absent, changes of other molecules, including SDHX, BRAF, NF1, K/N-RAS, and PIK3CA genes, may be involved [14]. Pathological diagnosis is similar in China and Europe. In the Chinese GIST guidelines, the algorithm of the pathological diagnosis is presented as shown in figure 1. IHC staining of GIST is recommended using 
a panel with five markers including CD117, DOG1, CD34, succinate dehydrogenase $\mathrm{B}$ (SDHB), and Ki67. SDHA markers could be added as appropriate $[5,15,16]$.

The molecular detection of GISTs is very important, and is helpful in the diagnosis of some difficult cases, the prediction of the therapeutic effect of molecular targeted drugs, and the guidance of clinical treatment. The Chinese guideline recommends that molecular detection should be carried out under the following circumstances:

- performing KIT or PDGFR $\alpha$ mutational analysis to identify the diagnosis of GIST in difficult cases; intending to use molecular targeted therapy preoperatively;

- all the cases initially diagnosed as recurrent or metastatic GIST and with molecular targeted therapy considered;

- the risk of recurrence of the primary resected GIST is moderate or high and the molecular targeted therapy is considered; to identify wild-type GIST;

- for differential diagnosis of synchronous and metachronous multiple GIST; or when the secondary resistance occurs with available specimens.

Detection of gene mutations should include at least exon 9, 11, 13, and 17 of the KIT gene and exons 12 and 18 of the PDGFR $\alpha$ gene. For patients with secondary resistance, exon 14 and 18 of the c-kit gene should also be included. The primary mutation of the KIT gene includes a variety of types of mutations and deletion mutation accounts for approximately $50 \%$ of the cases, in which codon 557-558 deletions lead to worse biological behavior than non-deletion mutations. They are also associated with a shorter effective imatinib treatment period. Identifying which types of c-kit exon 11 mutation provide information on the tumor biological behavior and therapeutic strategy decision for the individual patient is very important [17]. Therefore, the Chinese GIST guidelines suggest that a description of each type of exon 11 mutations of a GIST should be included in the molecular examination report.

\section{Risk Assessment of Primary Tumors}

The recurrence risk assessment system for the primary GIST after complete resection includes the modified National Institutes of Health (NIH) classification system (2006 [18]), the World Health Organization (WHO) TNM classification system (2013), the American Forces Institute of Pathology (AFIP) criteria, and the NCCN Guideline biological behavior predictor system (second version from 2016), prognostic contour map, and nomogram [19-22].

Among the major risk classification and staging systems, the AFIP criteria are indicated to accurately predict recurrence after complete surgery and are recommended by the NCCN and ESMO guidelines $[23,24]$. In view of the simplicity in application, however, the Chinese guideline recommends the modified NIH classification, which may be more suitable for the Asian population to select GIST patients who may have benefits from adjuvant therapy [25]. While there is still no perfect evaluation system, each center should combine the risk assessment with different circumstances. In the case of mitotic count, both guidelines recommend that mitosis should be expressed with a denominator of $5 \mathrm{~mm}^{2}$ because of different fields of view among microscopes. The existing evaluation system in China uses 50 high power fields (HPF) but the microscope eyepiece used by each center is different.

\section{Analysis of Prognostic Factors in Chinese Patients}

In an analysis by the China Gastrointestinal Stromal Tumor Study Group of prognostic factors of GISTs in 2,570 patients in a group of high-volume centers, the patient characteristics were quite typical compared to Western countries [26]. Of the included patients, 1,375 (53.5\%) were male, and the patient age range was 18-95 (median 58) years. The tumors were mostly found in the stomach (64.5\%), small intestine (25.1\%), and colorectal region (5.1\%). At the time of diagnosis, the median tumor size was $4.0 \mathrm{~cm}$. According to the modified NIH classification, 21.9, 28.9, 14.1, and $35.1 \%$ were very low-, low-, intermediate- and high-risk tumors, respectively. The rate of positivity was $96.4 \%$ for c-Kit, $87.1 \%$ for CD34, and $96.9 \%$ for delay of germination 1 (DOG-1). Multivariate analysis showed that age, tumor size, mitotic index, tumor site, occurrence of curative resection, and postoperative imatinib were independent prognostic factors. Furthermore, we found that highrisk patients benefited significantly from postoperative imatinib ( $\mathrm{p}$ $<0.001)$, whereas intermediate-risk patients did not $(\mathrm{p}=0.954)$ [26].

A subanalysis by the same group tried to establish the Ki-67 labelling index (LI) as a prognosticator in resected patients [27]. Multivariate analysis showed that Ki-67 LI was a significant predictor of overall survival (OS) (hazard ratio (HR) 1.793; 95\% confidence interval (CI) 1.240-2.593; $\mathrm{p}=0.002$ ). When stratified by a modified NIH classification, it was still independently associated with OS in high-risk and non-high-risk patients $(\mathrm{p}=0.001$ and $\mathrm{p}=$ 0.055 , respectively). Of note, the prognostic significance of Ki-67 LI was also maintained when stratified by tumor size, mitotic index, tumor site, and histological subtype (all $\mathrm{p}$ values $<0.05$ ). In addition, high-risk patients with Ki-67 LI $>6 \%$ exhibited a significantly lower OS rate than those with Ki-67 LI $\leq 6 \%$ (53.6 vs. 88.7\%, respectively; $\mathrm{p}=0.001$ ).

Starting from this database, a novel prognostic score was tried to be established in order to select only very high-risk patients for adjuvant imatinib and to build a basis for longer treatment duration [28]. Multivariate analysis revealed that only Ki-67 LI and mitotic index were independent prognostic factors of OS. For the two tumor-related pathological factors, Ki-67 LI > 7\% and the mitotic index $\geq 7 / 50 \mathrm{HPF}$ were allocated 1 point each. The total score was defined as the Pathological Prognostic Score (PPS). When Ki-67 LI and mitotic index were replaced by PPS, a multivariate analysis still identified PPS as an independent predictor of OS (HR 2.719; 95\% CI 1.309-5.650; $\mathrm{p}=0.007$ ). Patients with a PPS of 0,1 , or 2 had a 5 -year survival of $91.8,79.8$, and $51.0 \%$, respectively $(\mathrm{p}=0.001)$. Furthermore, an elevated PPS (PPS $=2$ ) was associated with larger 
tumor size, non-stomach tumor, and open resection (all p < 0.05). This PPS independently predicted postoperative survival in highrisk GISTs, and it might facilitate the selection of appropriate treatment strategy for these patients [28].

A very interesting approach concerns the prediction roles of clinical plus imaging features and multidetector computed tomography (MDCT) texture analysis in the preoperative risk classification of small bowel GISTs [29]. In a study on 213 patients, the model of clinical plus imaging findings showed an area under receiver operating characteristic curve (AUC) of 92.0\%. The AUC of the model combined clinical plus imaging features and MDCT texture analysis was $94.3 \%$, which was significantly higher than the AUC of the clinical imaging model $(\mathrm{p}=0.042)$.

\section{Surgical Treatment of GISTs}

Surgical resection of GISTs should be performed when complete removal can be achieved without affecting the function of the relevant organ. Routine biopsy is not recommended for most patients with resectable GIST. If preoperative therapy is necessary, biopsy should be performed. The biopsy should be carried out very carefully because inappropriate biopsy may cause tumor rupture, bleeding, and an increased risk of tumor dissemination. The methods of biopsy include: EUS-guided fine needle aspiration (EUS-FNA), core needle biopsy, endoscopic biopsy, transrectal or transvaginal needle aspiration biopsy, and intraoperative frozen biopsy. EUS-FNA is most recommended in the Chinese guideline because of the low probability of intraluminal implantation [30]. However, it is limited to the lumen of the digestive tract where EUS is available, and the pathological diagnosis is sometimes difficult because of the small amount of tissue obtained. Western guidelines recommend fine needle biopsy through the abdominal wall based on the results from retrospective studies (SSG-AIO XVIII), indicating that such biopsies did not increase the recurrent risk when appropriate surgery and/or medical therapy were done after biopsy [31].

Open or laparoscopic surgical R0 resections are the only potentially curative treatment for localized and potentially resectable GISTs. Given the fact that GISTs rarely have lymph node metastasis, routine dissection of regional lymph nodes is not needed. If enlarged lymph nodes were observed and lymphatic metastasis was suspected during operation, SDH-deficient GISTs should be considered and the enlarged lymph nodes should be dissected. The objective of operation is to achieve R0 resection as far as possible [16]. In the case of R1 resection and positive microscopic margin, adjuvant targeted therapy is recommended as there is no evidence that re-operation has a prognostic benefit.

The indications for laparoscopic surgery have been expanding in recent years. Laparoscopic resection can be performed in an experienced medical center according to the location and size of the tumor. Lesions $<5 \mathrm{~cm}$ in diameter located in favorable anatomic sites, such as the greater curvature or anterior wall of the gastric body and fundus, can be considered by the laparoscopic method. The significance of laparoscopic operation for jejunum and ileum GISTs is mainly to explore and locate the lesion. In addition, small GISTs in the upper rectum can also be considered with laparoscopic resection. If the large-sized tumor requires a larger abdominal incision to complete the removal, laparoscopic surgery is not recommended. Since tumor rupture is an independent adverse prognostic factor, surgery should follow the principle of 'no touch, less compression' and must use an 'extract bag' to avoid tumor rupture and spillage [32-34]. Since tumor rupture is the most ominous prognostic factor, most ruptured GISTs may have recurrences during follow-up [35]. Thus, all guidelines suggest imatinib adjuvant therapy for GIST patients with tumor rupture, and some experts may consider that these patients should receive adjuvant therapy for much longer than 3 years [36]. Hence, the Chinese guidelines indicate that further investigations are required to establish an optimized surveillance schedule with computed tomography (CT) for Asian GIST patients. The most common sites of metastasis after operation are the peritoneum and the liver, so abdominal and pelvic enhanced $\mathrm{CT}$ or magnetic resonance imaging (MRI) scan is recommended as a routine follow-up. Intermediate and high-risk patients should undergo CT or MRI examinations every 3 months for 3 years, then every 6 months until the 5 th year, and once per year thereafter. Low-risk patients should undergo CT or MRI examination every 6 months for 5 years.

\section{Molecular Targeted Drug Therapy in GISTs}

For medical treatment, the Asian and Western guidelines are very similar in recommending first-line imatinib, second-line sunitinib, and third-line regorafenib, as described above $[3,4,6,11]$. However, most of the evidence supporting these recommendations has been established by clinical trials conducted in the Western countries, and Asian GIST patients may still require their own evidence for some aspects, such as dose optimization. Whether GISTs with c-kit exon 9 mutation and wild-type GISTs could benefit from adjuvant therapy is controversial; there is still an insufficient amount of evidence because the relevant studies are based on small sample size, which cannot be used to evaluate treatment indications. Further clinical studies are needed [37].

For generalized progression of disease under standard dose imatinib therapy, it is recommended in China to shift to sunitinib or to escalate the dose of imatinib. Both continuous $37.5 \mathrm{mg} /$ day and $50.0 \mathrm{mg} /$ day $(4 / 2)$ programs are available for sunitinib therapy. Despite the lack of randomized controlled trials, sunitinib $37.5 \mathrm{mg} /$ day may have better efficacy and better tolerability. Data from Chinese studies shows that the benefit of sunitinib therapy in Chinese patients is better than that of Western patients, and the adverse reactions can be relieved by symptomatic treatment [38-40]. With regard to imatinib dose escalation, taking into account therapeutic tolerance, it is recommended to increase the imatinib dose to 600 $\mathrm{mg} /$ day first. The adverse effects of imatinib dose escalation can be relieved by symptomatic treatment [41]. 


\section{Conclusions}

Between China and Europe, there are some differences in the medical system, clinical practice, and disease profiles in oncology. Although the incidence of clinical GISTs appears to be similar between the two, the number of small GISTs treated by surgery seems to be higher in Asia. Major parts of the GIST guidelines are very similar between Asia and the West. However, there exist slight differences between the guidelines in the degree of recommendation, which may come from specific characteristics in clinical practice and available medicines. The ESMO clinical practice guidelines on GISTs describes the incidence of GISTs in Europe, the strategy to diagnose GISTs, the stage classification and risk assessment (does not recommend TNM classification), the staging procedure using CT, MRI and fluorodeoxyglucose-positron emission tomography scan, the treatment planning involving a multidisciplinary team for localized and metastatic disease, the response evaluation, and the optimal follow-up for different risk categories.

In the Chinese consensus guidelines for the diagnosis and management of GISTs, some aspects stand out. They recommend the minimal 3-year treatment with imatinib before and after surgery for high-risk GISTs [37]. Importantly, most clinical evidence in the GIST clinical guidelines has been established by clinical trials conducted in Western countries, and the number of clinical trials is still limited in Asia. This indicates that Asian GIST patients may have limited evidence based on their own data and may have limited access to new drugs after standard therapy. Finally, we may conclude that the GIST guidelines are well harmonized and that these guidelines will be of assistance to clinicians in Chinese and European hospitals to improve the diagnosis and management of GIST patients.

\section{Acknowledgment}

Funding: This work was supported by grants from the National Natural Science Foundation of China (81702303).

\section{Disclosure Statement}

The authors declare no conflict of interest.

\section{References}

1 Corless CL, Barnett CM, Heinrich MC: Gastrointestinal stromal tumours: origin and molecular oncology. Nat Rev Cancer 2011;11:865-878.

2 Fletcher CDM, Bridge JA, Hogendoorn PCW, et al: WHO Classification of Tumours of Soft Tissue and Bone, ed 4. Lyon, IARC Press, 2013, pp 164-167.

3 Hirota S, Isozaki K, Moriyama Y, et al: Gain-of-function mutations of c-kit in human gastrointestinal stromal tumors. Science 1998;279:577-580.

4 Koo DH, Ryu MH, Kim KM, et al: Asian Consensus Guidelines for the Diagnosis and Management of Gastrointestinal Stromal Tumor. Cancer Res Treat 2016; 48:1155-1166.

5 Li J, Ye Y, Wang J, et al: Chinese consensus guidelines for diagnosis and management of gastrointestinal stromal tumor. Chin J Cancer Res 2017;29:281-293.

6 Nishida T, Hirota S, Yanagisawa A, et al: Clinical practice guidelines for gastrointestinal stromal tumor (GIST) in Japan: English version. Int J Clin Oncol 2008;13:416-430.

7 Soreide K, Sandvik OM, Soreide JA, Giljaca V, Jureckova A, Bulusu VR: Global epidemiology of gastrointestinal stromal tumours (GIST): a systematic review of population-based cohort studies. Cancer Epidemiol 2016;40:39-46.

8 Raut CP: Tumor biological aspects of epithelial versus mesenchymal tumors of the gastrointestinal tract. Visc Med 2018;34:DOI: 10.1159/000493474.

9 Nishida T, Blay JY, Hirota S, Kitagawa Y, Kang YK The standard diagnosis, treatment, and follow-up of gastrointestinal stromal tumors based on guidelines. Gastric Cancer 2016;19:3-14.

10 Liu Q, Kong F, Zhou J, Dong M, Dong Q: Management of hemorrhage in gastrointestinal stromal tumors: a review. Cancer Manag Res 2018;10:735-743.

11 Ba MC, Qing SH, Huang XC, Wen Y, Li GX, Yu J: Diagnosis and treatment of small intestinal bleeding: retrospective analysis of 76 cases. World J Gastroenterol 2006;12:7371-7374.
Casali PG, Abecassis N, Aro HT, et al: Gastrointestinal stromal tumours: ESMO-EURACAN Clinical Practice Guidelines for diagnosis, treatment and follow-up. Ann Oncol 2018;29(suppl 4):iv267.

13 Wang M, Xu J, Zhang Y, et al: Gastrointestinal stromal tumor: 15-years' experience in a single center. BMC Surg 2014;14:93.

14 Hou YY, Lu SH, Zhou Y, et al: Predictive values of clinical and pathological parameters for malignancy of gastrointestinal stromal tumors. Histol Histopathol 2009;24:737-747.

15 Italiano A, Chen CL, Sung YS, et al: SDHA loss of function mutations in a subset of young adult wildtype gastrointestinal stromal tumors. BMC Cancer 2012;12:408.

16 Doyle LA, Nelson D, Heinrich MC, Corless CL, Hornick JL: Loss of succinate dehydrogenase subunit $\mathrm{B}$ (SDHB) expression is limited to a distinctive subset of gastric wild-type gastrointestinal stromal tumours: a comprehensive genotype-phenotype correlation study. Histopathology 2012;61:801-809.

17 Wang M, Xu J, Zhao W, et al: Prognostic value of mutational characteristics in gastrointestinal stromal tumors: a single-center experience in 275 cases. Med Oncol 2014;31:819.

18 Miettinen M, Lasota J: Gastrointestinal stromal tumors: pathology and prognosis at different sites. Semin Diagn Pathol 2006;23:70-83.

19 Joensuu H: Risk stratification of patients diagnosed with gastrointestinal stromal tumor. Hum Pathol 2008; 39:1411-1419.

20 Joensuu H, Vehtari A, Riihimaki J, et al: Risk of recurrence of gastrointestinal stromal tumour after surgery: an analysis of pooled population-based cohorts. Lancet Oncol 2012;13:265-274.

21 Gold JS, Gonen M, Gutierrez A, et al: Development and validation of a prognostic nomogram for recurrencefree survival after complete surgical resection of localised primary gastrointestinal stromal tumour: a retrospective analysis. Lancet Oncol 2009;10:1045-1052.
22 Rossi S, Gasparotto D, Miceli R, et al: KIT, PDGFRA, and BRAF mutational spectrum impacts on the natural history of imatinib-naive localized GIST: a populationbased study. Am J Surg Pathol 2015;39:922-930.

23 Goh BK, Chow PK, Yap WM, et al: Which is the optimal risk stratification system for surgically treated localized primary GIST? Comparison of three contemporary prognostic criteria in 171 tumors and a proposal for a modified Armed Forces Institute of Pathology risk criteria. Ann Surg Oncol 2008;15:2153-2163.

24 Bischof DA, Kim Y, Behman R, et al: A nomogram to predict disease-free survival after surgical resection of GIST. J Gastrointest Surg 2014;18:2123-2129.

25 Fletcher CD, Berman JJ, Corless C, et al: Diagnosis of gastrointestinal stromal tumors: a consensus approach. Hum Pathol 2002;33:459-465.

26 Liu X, Qiu H, Zhang P, et al: Prognostic factors of primary gastrointestinal stromal tumors: a cohort study based on high-volume centers. Chin J Cancer Res 2018;30:61-71.

27 Liu X, Qiu H, Zhang P, et al: Ki-67 labeling index may be a promising indicator to identify 'very high-risk' gastrointestinal stromal tumor: a multicenter retrospective study of 1022 patients. Hum Pathol 2018;74: $17-24$.

28 Liu X, Qiu H, Wu Z, et al: A novel Pathological Prognostic Score (PPS) to identify 'very high-risk' patients: a multicenter retrospective analysis of 506 patients with high risk gastrointestinal stromal tumor (GIST). J Gastrointest Surg 2018;DOI: 10.1007/s11605-0183799-5.

29 Yan J, Zhao X, Han S, Wang T, Miao F: Evaluation of clinical plus imaging features and multidetector computed tomography texture analysis in preoperative risk grade prediction of small bowel gastrointestinal stromal tumors. J Comput Assist Tomogr 2018;42:714720 . 
30 Dumonceau JM, Polkowski M, Larghi A, et al: Indications, results, and clinical impact of endoscopic ultrasound (EUS)-guided sampling in gastroenterology: European Society of Gastrointestinal Endoscopy (ESGE) Clinical Guideline. Endoscopy 2011;43:897912.

-31 Eriksson M, Reichardt P, Sundby Hall K, et al: Needle biopsy through the abdominal wall for the diagnosis of gastrointestinal stromal tumour - does it increase the risk for tumour cell seeding and recurrence? Eur J Cancer 2016;59:128-133.

32 Ronellenfitsch U, Staiger W, Kähler G, Strobel P, Schwarzbach M, Hohenberger P: Perioperative and oncological outcome of laparoscopic resection of gastrointestinal stromal tumour (GIST) of the stomach. Diagn Ther Endosc 2009;2009:286138.
Huang CM, Chen QF, Lin JX, et al: Can laparoscopic surgery be applied in gastric gastrointestinal stromal tumors located in unfavorable sites?: a study based on the NCCN guidelines. Medicine (Baltimore) 2017; 96:e6535.

34 Feng X, Li R, Zhang P, et al: Current status of surgical treatment of gastric gastrointestinal tumors: a national multi-center retrospective study (Article in Chinese). Zhonghua Wei Chang Wai Ke Za Zhi 2016;19:1258 1264.

35 Hohenberger P, Ronellenfitsch U, Oladeji O, Pink D, Krefter C, Ströbel P, et al. Pattern of recurrence in patients with ruptured primary Gastro-Intestinal Stromal Tumour (GIST). Br J Surg. 2010;97(12):1854-9.

36 Joensuu H, Hohenberger P, Corless CL: Gastrointestinal stromal tumour. Lancet. 2013;382(9896):973-83.

37 Joensuu H, Eriksson M, Sundby Hall K, et al: One vs three years of adjuvant imatinib for operable gastrointestinal stromal tumor: a randomized trial. JAMA 2012;307:1265-1272.
38 Li J, Gong JF, Li J, Gao J, Sun NP, Shen L: Efficacy of imatinib dose escalation in Chinese gastrointestinal stromal tumor patients. World J Gastroenterol 2012; 18:698-703.

39 Li J, Gao J, Hong J, Shen L: Efficacy and safety of sunitinib in Chinese patients with imatinib-resistant or -intolerant gastrointestinal stromal tumors. Future Oncol 2012;8:617-624.

40 Demetri GD, van Oosterom AT, Garrett CR, et al: Efficacy and safety of sunitinib in patients with advanced gastrointestinal stromal tumour after failure of imatinib: a randomised controlled trial. Lancet 2006; 368:1329-1338.

41 Zalcberg JR, Verweij J, Casali PG, et al: Outcome of patients with advanced gastro-intestinal stromal tumours crossing over to a daily imatinib dose of $800 \mathrm{mg}$ after progression on $400 \mathrm{mg}$. Eur J Cancer 2005;41:17511757. 